\title{
Nuclear receptors: emerging drug targets for parasitic diseases
}

\author{
Zhu Wang, ${ }^{1}$ Nathaniel E. Schaffer, ${ }^{1}$ Steven A. Kliewer, ${ }^{1,2}$ and David J. Mangelsdorf',3 \\ 'Department of Pharmacology, ${ }^{2}$ Department of Molecular Biology, and ${ }^{3}$ Howard Hughes Medical Institute, University of Texas Southwestern Medical Center, Dallas, Texas, USA
}

\begin{abstract}
Parasitic worms infect billions of people worldwide. Current treatments rely on a small group of drugs that have been used for decades. A shortcoming of these drugs is their inability to target the intractable infectious stage of the parasite. As wellknown therapeutic targets in mammals, nuclear receptors have begun to be studied in parasitic worms, where they are widely distributed and play key roles in governing metabolic and developmental transcriptional networks. One such nuclear receptor is DAF-12, which is required for normal nematode development, including the all-important infectious stage. Here we review the emerging literature that implicates DAF-12 and potentially other nuclear receptors as novel anthelmintic targets.
\end{abstract}

\section{Introduction}

One of the most prevalent classes of parasitic organisms is the helminth (parasitic worms), which collectively infect more than 1 billion people worldwide and cause a range of maladies including malnutrition, growth and mental retardation, disfigurement and physical disabilities, and death (1). Helminths that infect humans are generally considered to comprise two major phyla: Nematoda (round worms), both soil-transmitted and filarial; and Platyhelminthes (flatworms), both flukes and tapeworms. Parasitic worms represent one of the most successful and diverse extant forms of life. For example, of the 40,000 or more presumed nematode species, it is estimated that over half are parasitic (2). There also are believed to be more than 15,000 species of flatworm parasites. Mammalian parasites that are transmitted orally or through the skin from food or soil contaminated with eggs or young larvae usually colonize host intestines. Filarial parasites that are transmitted from biting insect vectors such as black flies or mosquitos enter the definitive host when the vector takes a blood meal. Filarial parasites infect a variety of different human tissues, with tissue specificity determined by the individual species (1). Unfortunately, few therapies exist to treat these parasites, and the long history of extensive use of anthelmintic drugs has resulted in a growing rate of resistance, which is now pervasive in nematode parasites infecting livestock (3). In the absence of alternative therapeutic strategies (e.g., vaccines), novel drugs to treat these infections are urgently needed.

Nuclear receptors are ligand-gated transcription factors that regulate diverse biological processes, including metabolism, development, and reproduction (4). Because of the lipophilic nature of their ligands and their ability to modulate the expression of multi-

Authorship note: Z. Wang and N.E. Schaffer are co-first authors.

Conflict of interest: D.J. Mangelsdorf is on the scientific advisory board of Metacrine Inc. and is a consultant to Reset Therapeutics. S.A. Kliewer owns stock in Intercept Pharmaceuticals Inc.

Reference information: / Clin Invest. 2017;127(4):1165-1171.

https://doi.org/10.1172/JCl88890. ple genes within the same pathway, nuclear receptors have become attractive targets for the development of orally available small-molecule drugs (4). Importantly, nuclear receptors have been identified in all categories of helminths (see ref. 5 for a systematic review of nuclear receptors found in helminths). We propose that targeting nuclear receptors would provide a novel therapeutic strategy that is distinct from current anthelmintic drugs, which target tubulins (benzimidazoles), ion channels (ivermectin and diethylcarbamazine), or symbotic bacteria of parasites (doxycycline). Here we review the current data suggesting nuclear receptors may be effective drug targets for treating helminthic diseases.

\section{Nuclear receptors in parasitic nematodes}

Although nuclear receptors are found in all worm phyla, the most studied have been in nematodes. It is of particular interest that the free-living nematode Caenorhabditis elegans has 284 nuclear receptors (6), the most of any known species (n.b., humans are a distant second with 48). The expansion of nuclear receptors in nematodes suggests they subserve an important biological advantage. Indeed, in addition to their roles in development and reproduction, several of these receptors have been shown to play key roles in survival by adapting the worm to various environmental stresses. One of the most studied of these receptors is DAF-12.

The nuclear receptor DAF-12. DAF-12 was initially described in C. elegans (7, 8), which is closely related to several species of human parasitic nematodes and has been used for anthelmintic drug discovery (9). C. elegans is able to adjust its developmental fate based on its environment. Under favorable conditions, i.e., low population density, proper temperature, and abundant food, C. elegans undergoes a rapid development from egg to reproductive adult through four larval stages, called L1 to L4. Under unfavorable conditions, however, C. elegans arrests its normal growth and enters a metabolic and developmental diapause at the third larval stage, called L3 dauer (L3d). The dauer diapause permits the worm to survive, often for long periods of time, until favorable conditions return (10). The primary function of DAF-12 is to regulate the nematode's environment-directed larval development as a transcrip- 
A

Favorable conditions, e.g., presence of nutrients, host-specific environment

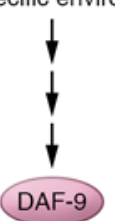

(Cytochrome P450) $\downarrow$

DAF-12 ligands

(dafachronic acids)

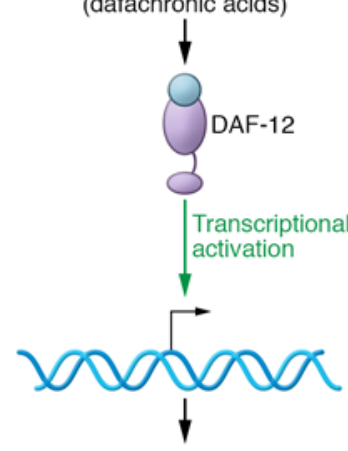

Reproductive maturity, host infection (increased metabolism/development)
B

Unfavorable conditions,
e.g., absence of nutrients, e.g., absence of nutrients,
preinfection environment
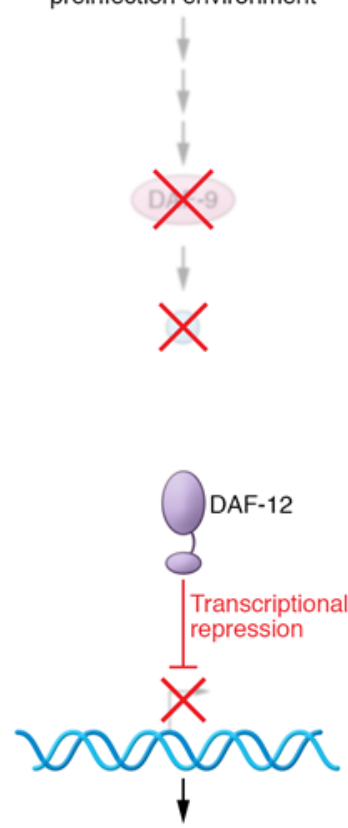

Dauer diapause, infectious stage (decreased metabolism, arrested development)
Figure 1. The DAF-12 signaling pathway. (A) Under favorable environmental conditions, the free-living nematode $C$. elegans stimulates the production of steroid-like hormones (i.e., DAs) that activate the DAF-12 nuclear receptor. DAF-12 activation results in transcription of a metabolic and development gene network that promotes reproductive maturity. (B) In unfavorable conditions when DAs are not synthesized, DAF-12 functions as a transcriptional repressor of this gene network that results in developmental arrest and entry into the dauer diapause. In helminthic nematodes, this same pathway is believed to govern the development of infectious larvae and the resumption of their development into reproductive maturity after entering their hosts. tional on/off switch (Figure 1). Favorable conditions induce synthesis of dafachronic acids (DAs), which are steroid hormones that bind to and activate DAF-12 $(11,12)$. The activated nuclear receptor induces expression of a gene network that coordinately promotes reproductive development and prevents dauer formation (13-15). In contrast, in unfavorable conditions DAs are not produced, and in this state the DAF-12 apo-receptor functions as a transcriptional repressor, preventing target gene expression and driving C. elegans into dauer diapause $(11,16)$. When favorable conditions return, ligand synthesis is resumed, the DAF-12 holo-receptor is activated, and the L3d larvae exit dauer diapause and resume reproductive development. Thus, DAF-12 is required for both entry into dauer (in the absence of ligand) and exit from dauer and resumption of reproductive development (in the presence of ligand) (Figure 1).

The rule of the infective third stage. An orthologous stage to dauer, called the infective third stage, exists in most parasitic nematodes (2). These parasites infect humans or other animals through a specialized infectious L3 (iL3) larval stage, which in monoxenous species (species that have a single host) is often transmitted through contact with contaminated soil (e.g., hookworms) and in heteroxenous species (species that have an intermediate host) is often transmitted by blood-sucking insect vectors (e.g., filarial worms). Homologs of DAF-12 have been isolated and characterized in several soil-borne parasites, including hookworms (Necator americanus, Ancylostoma ceylanicum, Ancylostoma caninum) and Strongyloides stercoralis, an incurable and often fatal parasite (17-19). Genome analysis also has revealed DAF-12 homologs in major species of filarial parasitic nematodes such as Brugia malayi (elephantiasis), Onchocerca volvulus (river blindness), and Loa loa (eye worm), and biochemical characterization of these DAF-12 homologs is underway.
Similar to their free-living counterparts, the iL3 larvae are developmentally arrested but resume their growth to reproductive adulthood after entering their definitive host via skin penetration. This so-called "rule of the infective third stage" (2) is analogous to the arrested L3d diapause stage of C. elegans (Figure 2). In the case of the parasite, the exit from iL3 occurs in the favorable conditions provided by the host, and similarly DAF- 12 has been shown to regulate this process $(15,17)$. In the absence of ligand, DAF-12 permits the formation of iL3 larvae, whereas upon entering the host (which presumably stimulates ligand synthesis) DAF-12 activation induces reproductive development. In S. stercoralis, treatment of infectious iL3 worms outside of their hosts with DAF-12 agonists initiates the resumption of feeding (19). Moreover, continual activation of DAF12 by pharmacologically administering DA to pre-infective larvae prevents the infective stage by inducing reproductive development of young larvae that would otherwise arrest their development as iL3s (19). Thus, the ligand status of DAF-12 appears to be essential for both initiating and establishing infection. The ability to interrupt the life cycle of the parasite at the infectious third stage by targeting DAF-12 activity is unprecedented and suggests an exciting potential anthelmintic therapeutic strategy.

DAF-12 mechanism of action. In C. elegans the mechanisms by which DAF-12 regulates larval development consist of transcriptional activation of gene programs controlling two basic processes. The first determines cell fate during larval development. This is exemplified by the development of seam cell lineages, which is regulated by the DAF-12 target genes mir-84 and mir-241, two let-7 family miRNAs $(13,20)$. During reproductive development, liganded DAF-12 induces mir-84 and mir-241 to inhibit the expression of a transcription factor, $h b l-1$, thereby directing the cells to an L3-specific fate $(13,20)$. During dauer formation 
A

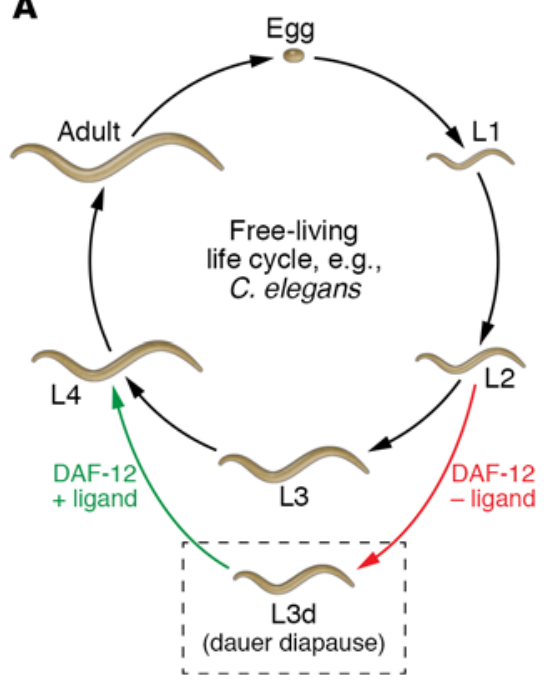

B

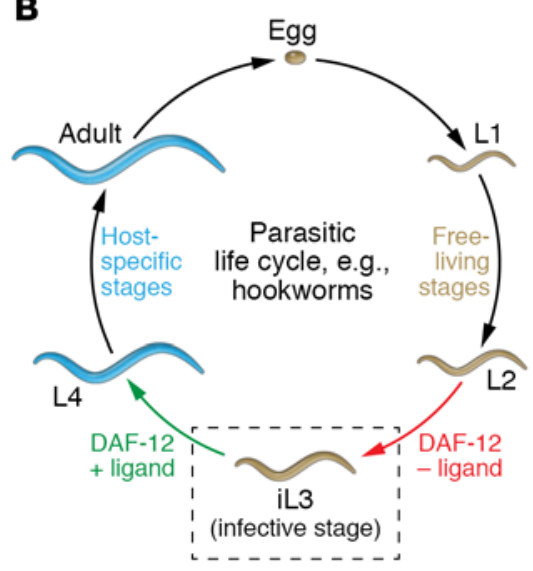

Figure 2. DAF-12 regulation of the iL3 stage as a novel therapeutic target. (A) In free-living nematodes, the decision to enter dauer diapause occurs at the third larval stage. Entry and exit from the L3d is determined by the presence or absence of DAF-12 ligands. (B) In parasitic nematodes, the iL3 stage is equivalent to the L3d of free-living nematodes, and this stage can be regulated by DAF-12 ligands. Although ligands for parasite DAF-12 receptors have not yet been identified, pharmacologic administration of DAs has been shown to prevent formation of iL3 larvae, thereby interrupting the parasite's life cycle. Thus, targeting DAF-12 in parasites may be an innovative new anthelmintic strategy. unliganded DAF-12 retains the seam cells in an L2-specific fate, which delays the switch to the L3-specific cell fate until reproductive development is resumed. The second process regulated by DAF- 12 is a coordinated shift in aerobic metabolism to meet the distinct energy requirements during larval development (15). In C. elegans, liganded DAF-12 upregulates a gene network that promotes aerobic metabolism of fat, allowing efficient production of sufficient energy for rapid reproductive growth. Unliganded DAF-12, however, limits fat consumption to a lower rate of anaerobic metabolism. This prevents the dauer larvae from prematurely exhausting energy stores and permits survival in unfavorable conditions.

The mechanistic details of DAF-12 regulation of larval development in parasites remain incompletely elucidated, but basic elements of this pathway appear to be conserved from C. elegans. In the soil-transmitted species S. stercoralis, liganded DAF-12 induces a variety of genes that are involved in fat utilization, and as in C. elegans, inhibition of aerobic fat utilization can inhibit the parasite's reproductive development (15). Although no let-7 family miRNAs exist in Strongyloides spp. (21), hbl-1 homologs have been identified (WormBase ID: SRAE_1000178600). Therefore, $h b l-1$ may also mediate DAF-12-regulated larval development in parasitic nematodes through a distinct mechanism from the let-7 family of miRNAs. In contrast, both let-7 family miRNAs and $h b l-1$ are conserved in filarial nematodes. Moreover, in Brugia pahangi, which causes a zoonotic filariasis, the increased expression of several let-7 homologs correlates well with iL3 exit from dauer-like diapause upon invasion of the host (22). Although the hypothesis remains to be tested, these let-7 homologs potentially mediate the control of developmental progression by DAF- 12 .

Besides impacting the development of young nematode larvae, DAF-12 can also affect longevity in adult nematodes. In the germline longevity pathway, DAs are synthesized to activate DAF12 in response to germline ablation and extend lifespan through the canonical longevity pathway regulated by the FOXO homolog DAF-16 (23). In this context, ligand activation of DAF-12 promotes DAF-16 activity by inducing the let-7 family of miRNAs that sup- press the DAF-16 inhibitory genes lin-14 and akt-1 (a PI3K homo$\log )$. The activated DAF-16 then extends lifespan by inducing longevity-associated target genes $(24,25)$. Long lifespans have been observed in many adult species of parasitic nematodes (26), including some of the most harmful species such as hookworm and filarial nematodes, which can live in the same host for several decades. Although the mechanistic understanding of this longevity is incompletely understood, the germline longevity pathway is likely to be conserved in parasitic nematodes. Prospectively, during aging as the germline cells are lost, DAF-12 and DAF-16 may be engaged to extend the lifespan. Both DAF-12 and DAF-16 are conserved in these parasitic nematodes, further suggesting the presence of a similar regulation of longevity in parasites.

Targeting DAF-12 receptors. The principle of targeting DAF12 to treat parasitic diseases relies on using exogenous ligands to interfere with the physiologic functions of the receptor. The ideal drugs should be DAF-12 modulators that target entry into and exit from the iL3 stage. One potential drug is $\Delta 7$-dafachronic acid $(\triangle 7-\mathrm{DA})$, which is the endogenous DAF-12 ligand in C. elegans and also activates some parasite DAF-12s, albeit with less potency and efficacy (19).

$S$. stercoralis is a parasite that has been used to test the therapeutic potential of DA-like drugs. This parasite has evolved two developmental strategies for establishing infections. In one pathway, newly hatched L1 larvae in the host intestine are excreted in the feces, and at ambient environmental temperatures these L1s develop into free-living adults (17). After one free-living life cycle, the second generation of these offspring develops into iL3 larvae that infect new hosts. In the second pathway, the newly hatched L1 larvae stay within the host and at $37^{\circ} \mathrm{C}$ establish a perennial autoinfection by developing directly into iL3 larvae that continually re-infect the host. The inability of current anthelmintic drugs (e.g., ivermectin, benzimidazoles) to target the latent autoinfective larvae is a major reason why these drugs are often ineffective at curing strongyloidiasis. In contrast, the use of DAF-12 ligands may be a more efficacious strategy for preventing autoinfection and eliminating the parasites. In proof-of-concept experiments in which young, post-free-living $S$. stercoralis larvae were treated with 
$\Delta 7-\mathrm{DA}$, the majority of larvae completely bypassed the iL3 stage (17) and instead were driven toward precocious, incomplete reproductive development and death. In addition, when post-parasitic L1 larvae freshly isolated from the host were cultured in host-like conditions (i.e., $37^{\circ} \mathrm{C}$ ) that promote development into autoinfective L3 larvae, treatment with $\Delta 7-\mathrm{DA}$ stimulated development into free-living adults and completely prevented the occurrence of any iL3 larvae (17). The efficacy of $\triangle 7$-DA under these conditions likely resulted from $\Delta 7$-DA acting as a partial agonist that prevents unliganded DAF-12 from causing iL3 developmental arrest. In contrast, administration of $\triangle 7-\mathrm{DA}$ to hosts infected with $S$. stercoralis might be expected to prevent autoinfection and interrupt normal reproductive development. Given the association between DAF12 signaling and longevity, disrupting DAF-12 signaling with selective DAF-12 modulators like $\Delta 7$-DA would be expected to have two beneficial therapeutic attributes: they would prevent immature worms from entering the infective stage (by preventing the "dauer" pathway) and shorten the lifespan of the adult parasites (by inhibiting the germline pathway). This latter effect might be of particular importance in eliminating the long-lived parasitic nematodes such as the filarial nematodes and hookworms.

Taken together, current research suggests that DAF-12 is a promising therapeutic target. DAF-12 controls important biological processes that are essential for parasite transmission, being required for both entry into and exit from the dauer-like iL3 stages. This dual functionality of DAF-12 provides two potential modes of therapeutic effect. Furthermore, DAF-12 homologs thus far have been identified in all parasitic nematodes that undergo an L3 developmental arrest, which indicates that a drug targeting the receptor would be broadly applicable. It is also noteworthy that the specificity of DA for DAF-12 binding and activation is sufficiently high to prevent cross-over activity with host nuclear receptors, which are only distantly related (11). Finally, the crystal structure of the DAF-12 ligand-binding domain has been solved for two parasitic species $(19,27)$, which could guide the discovery and optimization of new drugs. Importantly, the potential therapeutic benefit of targeting DAF-12 has already been validated in S. stercoralis.

Targeting DAF-12 ligand synthesis. In addition to targeting DAF12 , another potential therapeutic strategy is to target the biosynthetic pathway that generates the ligand as a means of preventing DAF-12 activation, thereby interrupting development and infection. In the free-living C. elegans, the key enzyme in this biosynthetic pathway is the cytochrome P450 DAF-9 $(11,28,29)$. Worms lacking DAF-9 are constitutive dauers (i.e., daf- 9 mutants permanently arrest as L3d larvae) but can be rescued to full reproductive adulthood via hormone replacement therapy with exogenously administered DAs $(11,30)$. Interestingly, DAF-9 homologs have not been identified in any parasitic nematode (our unpublished observation), suggesting that parasites lack the ability to synthesize their own DAF-12 ligands. This finding would explain why parasitic larvae behave like daf-9 mutants and must become iL3 larvae, even under conditions that might otherwise seem favorable. Furthermore, this brings up the intriguing possibility that the host is the source of the parasite's DAF-12 ligand, since it is only in the presence of the host that DAF-12 activation occurs. In support of this hypothesis, it is interesting that the mammalian P450, CYP27A1, is functionally orthologous to DAF-9 and can synthesize DA (and perhaps other, more potent parasite-specific DAF-12 ligands) from sterol precursors that are found only in C. elegans $(11,27)$. Thus, it is exciting to speculate that the biosynthetic pathway for the ligand might be targeted to inhibit DAF-12 activation in the host.

The nuclear receptor NHR-8. NHR-8 is another nuclear receptor that is conserved between C. elegans and parasitic nematodes (31). As revealed by studies in C. elegans, the central role of this nuclear receptor is to regulate dietary sterol utilization. Nematodes are cholesterol auxotrophs (32) and dietary cholesterol is the sole source of the sterol precursors for the ligands for DAF-12 (i.e., DAs) and perhaps other nuclear receptors. In C. elegans, disruption of NHR-8 leads to defects in DA synthesis and causes a constitutive dauer $(d a f-c)$ phenotype under stringent conditions such as high temperature or cholesterol restriction (31). NHR-8 likely regulates the transport of sterols between tissues where the DA biosynthetic enzymes are expressed, a process that is required for generating DA. Consistent with this hypothesis, NHR-8 induces the expression of the apolipoproteins vit-1 and vit-2, which are components of lipoprotein complexes that transport sterols (33, 34). Thus, inhibiting this pathway would be expected to disrupt DAF-12 signaling.

Although the mechanistic details of NHR-8 function are not yet clear, its ligand-binding domain appears dispensable, as determined by phenotypic analysis of different $n h r-8$ allelic mutants. Despite being observed in two null mutants, the daf-c phenotype is not seen in a truncated $n h r-8$ allele (ok186), which encodes an NHR-8 protein lacking the entire ligand-binding domain and a large portion of the hinge region (31). This suggests that the DNA-binding domain alone may be sufficient for NHR-8 function. Thus, it is not clear whether targeting NHR-8 with a ligand-like molecule would be a plausible strategy for treating parasitic infections. Nevertheless, it may become possible to target the upstream regulators or downstream effectors of NHR-8 once the pathway has been fully elucidated.

The nuclear receptors NHR-49 and NHR-80. In C. elegans there are 270 homologs of the mammalian nuclear receptor HNF4, making it the largest subfamily of nuclear receptors in any species and highlighting a rare natural gene amplification event within the animal kingdom. Studies in C. elegans (35-44) indicate that this family of nuclear receptors regulates lipid metabolism, similar to their homologs in flies and mammals $(45,46)$. The tremendous expansion of this nuclear receptor subfamily may reflect the need for C. elegans to sense and adapt to diverse environments.

In C. elegans ablation of the germline increases lifespan. Interestingly, one of these HNF4-like proteins, NHR-49, is required for the longevity effect. NHR-49 regulates genes involved in fatty acid metabolism and is required for germline-ablated worms to maintain their lipid reserves $(43,44,47,48)$. NHR-49 is also required for adult reproductive diapause, a starvation-induced mechanism that protects reproductive potential until nutrients become available (49). NHR-49 activity is regulated in part at the level of its expression. Upon germline removal, NHR-49 is upregulated in somatic tissues, which is essential for extending lifespan (47). However, other regulatory mechanisms must exist because during nutrient deprivation, when NHR-49 is functionally important, the expression of NHR49 is either unchanged (36) or only marginally increased (15). This suggests that activation of NHR-49 by a ligand may be important 
for its function. Although ligands for NHR-49 have not been identified, there is reason to believe that they may be fatty acids or related metabolites. Mammalian HNF $4 \alpha$ has been shown to bind fatty acids (50), and long-chain fatty acids can activate Drosophila HNF4 and mimic starvation-induced activity in vivo (45).

NHR-80 is another HNF4-like nuclear receptor that is required for the extended lifespan in germline-ablated C. elegans (37). NHR-80 expression is upregulated and accumulates in intestinal nuclei, where it appears to induce monodesaturation of fatty acids. NHR-80 also directly interacts with NHR-49 (41), and this heterodimer is believed to be ligand activated by specific unsaturated fatty acid metabolites that promote longevity (48).

NHR-49 and NHR-80 homologs have been found in S. stercoralis and the filarial nematodes $B$. malayi and $L$. loa, with over $80 \%$ and $60 \%$ sequence identities in the DNA- and ligand-binding domains, respectively (our unpublished observation). This suggests that the NHR-49/NHR-80 longevity pathway is conserved in parasitic nematodes and might be exploited therapeutically. Given their role in regulating lifespan, targeting these nuclear receptors might be particularly useful to control long-lived parasitic nematodes (26). Administration of compounds that antagonize NHR-49/NHR-80 activity might potentially interfere with their physiologic functions and block survival and reproduction of the parasites.

Ecdysone receptor homologs in filarial nematodes. Ecdysone receptors (EcRs) were first characterized in arthropods, where they control development and reproduction in response to molting hormones called ecdysteroids (51-53). EcR homologs have been identified in filarial nematodes including the dog heartworm Dirofilaria immitis (54) and the human parasite B. malayi (55), and ecdysteroids have been reported to activate the filarial EcRs (55). In B. malayi, 20-hydroxyecdysterone, a common ecdysteroid in insect species, can induce a transgenic reporter driven by EcR response elements. The EcR homologs in nematodes can also form heterodimers with RXR homologs in response to ecdysteroids, which is a prerequisite for activation of EcR in insects (55). However, the ecdysteroids tested thus far do not seem to be the physiologic ligands for the parasite EcR homologs. Ecdysteroids activate nematode EcRs at micromolar concentrations, which are much higher concentrations than the nematodes would be exposed to inside their insect vectors (56). Nevertheless, it is reasonable to speculate that EcR homologs are physiologically activated by ligands that are derived from insect ecdysteroids.

Despite the absence of identified endogenous ligands, the filarial EcRs would appear to be attractive drug targets for treating filariasis. In a B. malayi infection model in gerbils, 20-hydroxyecdysterone treatment eliminated adult worm burden in infected hosts (57), indicating that larval development was impaired. Two potent ecdysone analogs, muristerone $\mathrm{A}$ and ponasterone $\mathrm{A}$, can activate the B. malayi $\mathrm{EcR}$ at nanomolar concentrations and promote the females to expel immature eggs, embryos, and microfilariae in an in vitro assay (57). Thus, ectopic activation of the B. malayi EcR is likely to interfere with the development and reproduction of the parasites. Since muristerone A and ponasterone A are approximately 50 times more potent than 20-hydroxyecdysterone in activating B. malayi EcR (57), it will be interesting to test whether they can more efficiently eliminate the adult worm burden in the gerbil model.

\section{Nuclear receptors in parasitic flatworms}

Flukes cause one of the most devastating parasitic diseases, schistosomiasis, and infect over 200 million people worldwide. In humans, schistosomiasis is primarily caused by Schistosoma mansoni and Schistosoma japonicum (58). So far, 21 nuclear receptors have been found in $S$. mansoni and $S$. japonicum, but their functions are unknown (59). Homologs of several genes that mediate ecdysone responses in insects, including the ecdysone response genes E78 and FTZ-F1 and the heterodimeric partner of EcR, RXR, are also present in S. mansoni (59). Furthermore, S. mansoni synthesizes two ecdysteroids, ecdysone and 20-hydroxyecdysone, with peak production occurring during the liver stage, in which the parasite larvae develop into mature adults (60). However, to date no EcR homolog has been identified. These findings suggest that an ecdysteroid-nuclear receptor system may control the reproductive development of the parasites, although the identity of the nuclear receptor remains unknown.

S. mansoni E78 is a nuclear receptor homologous to the Drosophila ecdysone response gene $78(59,61)$. In Drosophila, E78 is essential for establishing germline stem cells and thus is crucial for fertility (62). In S. mansoni, the E78 homolog is highly expressed in the egg, miracidiae, and daughter sporocyst stages, which all occur in the intermediate host (usually snails) (61). This expression pattern indicates that E78 may play an important role in amplifying the parasite before it infects humans, the definitive host.

One unusual class of nuclear receptors found in S. mansoni is composed of three proteins called Sm2DBD-NR $\alpha, \operatorname{Sm} 2 \mathrm{DBD}-\mathrm{NR} \beta$, and Sm2DBD-NR $\gamma$, which atypically contain two DNA-binding domains linked to a single ligand-binding domain $(59,63,64)$. NHR-1, a nuclear receptor that is homologous to Sm2DBD-NR $\gamma$, has been identified in planarians, which are free-living relatives of S. mansoni. NHR-1 and Sm2DBD-NR $\gamma$ share $74 \%$ and $83 \%$ sequence identity in the two DNA-binding domains and $44 \%$ identity in the ligand-binding domain (65). In planarians, NHR-1 is required for the development of accessory reproductive organs and is important for the differentiation and maturation of germ cells (65). The functions of the Sm2DBD-NRs in S. mansoni remain to be determined, but they are differentially expressed in various developmental stages (64), which suggests that they may regulate parasite development and thus be therapeutic targets.

The study of nuclear receptors in tapeworms began only recently in Echinococcus multilocularis (66). The life cycle of E. multilocularis involves an intermediate host (a rodent) and a definitive host (a fox or dog). Three successive stages of larvae, named onchosphere, metacestode, and protoscolex, live in the intermediate host, and upon entering the definitive host, the protoscolex larvae develop into reproductive adults. Genome analyses have revealed 17 putative nuclear receptors in E. multilocularis, most of which are homologous to the nuclear receptors in S. mansoni (66), including one named EmNHR-1. Although this nuclear receptor is expressed in all larval stages, it is upregulated in metacestode and dormant protoscolex larvae, which live and arrest in the intermediate host. Thus, EmNHR-1 may be required for parasite development in the intermediate host. The tapeworm species that infect humans (e.g., pork and beef tapeworms) have a similar life cycle to E. multilocularis, and it will be interesting to explore the existence and function of EmNHR-1 homologs in these human parasites. 


\section{Summary}

Nuclear receptors are promising drug targets for treating parasitic diseases. They have been found in all parasitic helminths studied and, like their counterparts in higher animals, they control essential biological processes that govern metabolism, development, and reproduction. Therefore, modulating nuclear receptor signaling may provide exciting and novel therapeutic opportunities. Although nuclear receptors have begun to be studied in depth in their free-living relatives, there has been a paucity of research in helminths. Major limitations to advancing nuclear receptor biology in parasites include the obvious technical difficulties associated with working with parasites and their hosts and the absence of traditional genetic tools for parasitic worms. However, recent advances in technologies such as clustered regularly interspaced short palindromic repeats (CRISPR) may afford a significant opportunity to expand this line of research. Future work will also need to focus on characterizing the biosynthetic pathways that generate ligands of parasite nuclear receptors. Thus far, no endogenous nuclear receptor ligands have been identified in parasitic worms; however, the characterization of the DAF-12 ligands for the free-living nematode $C$. elegans provides a useful precedent and strategy for doing so. Finally, it will be crucial to develop synthetic ligands for parasite nuclear receptors that might be used both as tool compounds to interrogate their biological function and as candidate therapeutics.

\section{Acknowledgments}

This work was supported by NIH grant R33AI105856 (to DJM), the Robert A. Welch Foundation (grant I-1558 to SAK and grant I-1275 to DJM), and the Howard Hughes Medical Institute (grant to DJM).

Address correspondence to: Steven A. Kliewer or David J. Mangelsdorf, University of Texas Southwestern Medical Center, 6001 Forest Park Rd., Dallas, Texas 75390, USA. Phone: 214.645.6304; E-mail: steven.kliewer@utsouthwestern.edu (S.A. Kliewer). Phone: 214.645.5957; E-mail: davo.mango@utsouthwestern.edu (D.J. Mangelsdorf).
1. Hotez PJ, Brindley PJ, Bethony JM, King CH, Pearce EJ, Jacobson J. Helminth infections: the great neglected tropical diseases. J Clin Invest. 2008;118(4):1311-1321.

2. Anderson RC. Introduction. In: Anderson RC, ed. Nematode Parasites Of Vertebrates: Their Development And Transmission. 2nd ed. Oxfordshire, United Kingdom: CABI; 2000:1-18.

3. Prichard R. Anthelmintic resistance. Vet Parasitol. 1994;54(1-3):259-268.

4. Evans RM, Mangelsdorf DJ. Nuclear receptors, RXR, and the Big Bang. Cell. 2014;157(1):255-266.

5. Wu W, LoVerde PT. Nuclear hormone receptors in parasitic helminths. Mol Cell Endocrinol. 2011;334(1-2):56-66.

6. Antebi A. Nuclear hormone receptors in C. elegans (January 03, 2006). WormBook, ed. The C. elegans Research Community, WormBook, doi/10.1895/wormbook.1.64.1, http://www. wormbook.org.

7. Antebi A, Culotti JG, Hedgecock EM. daf-12 regulates developmental age and the dauer alternative in Caenorhabditis elegans. Development. 1998;125(7):1191-1205.

8. Antebi A, Yeh WH, Tait D, Hedgecock EM, Riddle DL. daf-12 encodes a nuclear receptor that regulates the dauer diapause and developmental age in C. elegans. Genes Dev. 2000;14(12):1512-1527.

9. Holden-Dye L, Walker RJ. Anthelmintic drugs and nematicides: studies in Caenorhabditis elegans (December 16, 2014). WormBook, ed. The C. elegans Research Community, WormBook, doi/10.1895/wormbook.1.143.2, http://www. wormbook.org.

10. Riddle DL, Albert PS. Genetic and environmental regulation of dauer larva development. In: C. elegans II. 2nd ed. Cold Spring Harbor, New York, USA: Cold Spring Harbor Laboratory Press; 1997:739-768.

11. Motola DL, et al. Identification of ligands for DAF12 that govern dauer formation and reproduction in C. elegans. Cell. 2006;124(6):1209-1223.

12. Mahanti P, et al. Comparative metabolomics reveals endogenous ligands of DAF-12, a nuclear hormone receptor, regulating C. elegans development and lifespan. Cell Metab. 2014;19(1):73-83.

13. Bethke A, Fielenbach N, Wang Z, Mangelsdorf DJ, Antebi A. Nuclear hormone receptor regulation of microRNAs controls developmental progression. Science. 2009;324(5923):95-98.

14. Hochbaum D, et al. DAF-12 regulates a connected network of genes to ensure robust developmental decisions. PLoS Genet. 2011;7(7):e1002179.

15. Wang $\mathrm{Z}$, et al. The nuclear receptor DAF-12 regulates nutrient metabolism and reproductive growth in nematodes. PLoS Genet. 2015;11(3):e1005027.

16. Ludewig AH, et al. A novel nuclear receptor/ coregulator complex controls C. elegans lipid metabolism, larval development, and aging. Genes Dev. 2004;18(17):2120-2133.

17. Albarqi MM, et al. Regulation of life cycle checkpoints and developmental activation of infective larvae in Strongyloides stercoralis by dafachronic acid. PLoS Pathog. 2016;12(1):e1005358.

18. Stoltzfus JD, Bart SM, Lok JB. cGMP and NHR signaling co-regulate expression of insulin-like peptides and developmental activation of infective larvae in Strongyloides stercoralis. PLoS Pathog. 2014;10(7):e1004235.

19. Wang $Z$, et al. Identification of the nuclear receptor DAF-12 as a therapeutic target in parasitic nematodes. Proc Natl Acad Sci US A. 2009;106(23):9138-9143.

20. Hammell CM, Karp X, Ambros V. A feedback circuit involving let-7-family miRNAs and DAF-12 integrates environmental signals and developmental timing in Caenorhabditis elegans. Proc Natl Acad Sci U S A. 2009;106(44):18668-18673.

21. Stoltzfus JD, Minot S, Berriman M, Nolan TJ, Lok JB. RNAseq analysis of the parasitic nematode Strongyloides stercoralis reveals divergent regulation of canonical dauer pathways. PLoS Negl Trop Dis. 2012;6(10):e1854.

22. Winter AD, et al. A novel member of the let-7 microRNA family is associated with develop- mental transitions in filarial nematode parasites. BMC Genomics. 2015;16:331.

23. Shen Y, Wollam J, Magner D, Karalay O, Antebi A. A steroid receptor-microRNA switch regulates life span in response to signals from the gonad. Science. 2012;338(6113):1472-1476.

24. Murphy CT, et al. Genes that act downstream of DAF-16 to influence the lifespan of Caenorhabditis elegans. Nature. 2003;424(6946):277-283.

25. Tepper RG, Ashraf J, Kaletsky R, Kleemann G, Murphy CT, Bussemaker HJ. PQM-1 complements DAF-16 as a key transcriptional regulator of DAF-2-mediated development and longevity. Cell. 2013;154(3):676-690.

26. Gems D. Longevity and ageing in parasitic and free-living nematodes. Biogerontology. 2000;1(4):289-307.

27. Zhi X, et al. Structural conservation of ligand binding reveals a bile acid-like signaling pathway in nematodes. J Biol Chem. 2012;287(7):4894-4903.

28. Gerisch B, Weitzel C, Kober-Eisermann C, Rottiers V, Antebi A. A hormonal signaling pathway influencing C. elegans metabolism, reproductive development, and life span. Dev Cell. 2001;1(6):841-851.

29. Jia K, Albert PS, Riddle DL. DAF-9, a cytochrome $\mathrm{P} 450$ regulating $\mathrm{C}$. elegans larval development and adult longevity. Development. 2002;129(1):221-231.

30. Sharma KK, Wang Z, Motola DL, Cummins CL, Mangelsdorf DJ, Auchus RJ. Synthesis and activity of dafachronic acid ligands for the C. elegans DAF-12 nuclear hormone receptor. Mol Endocrinol. 2009;23(5):640-648.

31. Magner DB, et al. The NHR-8 nuclear receptor regulates cholesterol and bile acid homeostasis in C. elegans. Cell Metab. 2013;18(2):212-224.

32. Kurzchalia TV, Ward S. Why do worms need cholesterol? Nat Cell Biol. 2003;5(8):684-688.

33. Spieth J, Blumenthal T. The Caenorhabditis elegans vitellogenin gene family includes a gene encoding a distantly related protein. Mol Cell Biol. 1985;5(10):2495-2501. 
34. Spieth J, Denison K, Kirtland S, Cane J, Blumenthal $\mathrm{T}$. The $\mathrm{C}$. elegans vitellogenin genes: short sequence repeats in the promoter regions and homology to the vertebrate genes. Nucleic Acids Res. 1985;13(14):5283-5295.

35. Antebi A. Nuclear receptor signal transduction in C. elegans (June 9, 2015). WormBook, ed. The C. elegans Research Community, WormBook, doi/10.1895/wormbook.1.64.2, http://www. wormbook.org.

36. Goh GY, et al. The conserved Mediator subunit MDT- 15 is required for oxidative stress responses in Caenorhabditis elegans. Aging Cell. 2014;13(1):70-79.

37. Goudeau J, Bellemin S, Toselli-Mollereau E, Shamalnasab M, Chen Y, Aguilaniu H. Fatty acid desaturation links germ cell loss to longevity through NHR-80/HNF4 in C. elegans. PLoS Biol. 2011;9(3):e1000599.

38. Heestand BN, et al. Dietary restriction induced longevity is mediated by nuclear receptor NHR-62 in Caenorhabditis elegans. PLoS Genet. 2013;9(7):e1003651.

39. Liang B, Ferguson K, Kadyk L, Watts JL. The role of nuclear receptor NHR-64 in fat storage regulation in Caenorhabditis elegans. PLoS One. 2010;5(3):e9869.

40. Noble T, Stieglitz J, Srinivasan S. An integrated serotonin and octopamine neuronal circuit directs the release of an endocrine signal to control C. elegans body fat. Cell Metab. 2013;18(5):672-684.

41. Pathare PP, Lin A, Bornfeldt KE, Taubert S, Van Gilst MR. Coordinate regulation of lipid metabolism by novel nuclear receptor partnerships. PLoS Genet. 2012;8(4):e1002645.

42. Taubert S, Van Gilst MR, Hansen M, Yamamoto KR. A Mediator subunit, MDT-15, integrates regulation of fatty acid metabolism by NHR-49-dependent and -independent pathways in C. elegans. Genes Dev. 2006;20(9):1137-1149.

43. Van Gilst MR, Hadjivassiliou H, Jolly A, Yamamoto KR. Nuclear hormone receptor NHR-49 controls fat consumption and fatty acid composition in C. elegans. PLoS Biol. 2005;3(2):e53.

44. Van Gilst MR, Hadjivassiliou H, Yamamo- to KR. A Caenorhabditis elegans nutrient response system partially dependent on nuclear receptor NHR-49. Proc Natl Acad Sci U S A. 2005;102(38):13496-13501.

45. Palanker L, Tennessen JM, Lam G, Thummel CS. Drosophila HNF4 regulates lipid mobilization and $\beta$-oxidation. Cell Metab. 2009;9(3):228-239.

46. Martinez-Jimenez CP, Kyrmizi I, Cardot P, Gonzalez FJ, Talianidis I. Hepatocyte nuclear factor $4 \alpha$ coordinates a transcription factor network regulating hepatic fatty acid metabolism. Mol Cell Biol. 2010;30(3):565-577.

47. Ratnappan R, et al. Germline signals deploy NHR-49 to modulate fatty-acid $\beta$-oxidation and desaturation in somatic tissues of C. elegans. PLoS Genet. 2014;10(12):e1004829.

48. Folick A, et al. Aging. Science. 2015;347(6217):83-86.

49. Angelo G, Van Gilst MR. Starvation protects germline stem cells and extends reproductive longevity in C. elegans. Science. 2009;326(5955):954-958.

50. Dhe-Paganon S, Duda K, Iwamoto M, Chi YI, Shoelson SE. Crystal structure of the HNF $4 \alpha$ ligand binding domain in complex with endogenous fatty acid ligand. J Biol Chem. 2002;277(41):37973-37976.

51. Koelle MR, Talbot WS, Segraves WA, Bender MT, Cherbas P, Hogness DS. The Drosophila EcR gene encodes an ecdysone receptor, a new member of the steroid receptor superfamily. Cell. 1991;67(1):59-77.

52. Yao TP, Segraves WA, Oro AE, McKeown M, Evans RM. Drosophila ultraspiracle modulates ecdysone receptor function via heterodimer formation. Cell. 1992;71(1):63-72.

53. Yao TP, et al. Functional ecdysone receptor is the product of EcR and Ultraspiracle genes. Nature. 1993;366(6454):476-479.

54. Shea C, Richer J, Tzertzinis G, Maina CV. An EcR homolog from the filarial parasite, Dirofilaria immitis requires a ligand-activated partner for transactivation. Mol Biochem Parasitol. 2010;171(2):55-63.

55. Tzertzinis G, et al. Molecular evidence for a functional ecdysone signaling system in Brugia malayi. PLoS Negl Trop Dis. 2010;4(3):e625.
56. Margam VM, Gelman DB, Palli SR. Ecdysteroid titers and developmental expression of ecdysteroid-regulated genes during metamorphosis of the yellow fever mosquito, Aedes aegypti (Diptera: Culicidae). JInsect Physiol. 2006;52(6):558-568.

57. Mhashilkar AS, et al. Identification of ecdysone hormone receptor agonists as a therapeutic approach for treating filarial infections. PLoS Negl Trop Dis. 2016;10(6):e0004772.

58. Gryseels B, Polman K, Clerinx J, Kestens L. Human schistosomiasis. Lancet. 2006;368(9541):1106-1118.

59. Wu W, LoVerde PT. Nuclear hormone receptors in parasitic helminths. Mol Cell Endocrinol. 2011;334(1-2):56-66.

60. Nirde P, Torpier G, De Reggi ML, Capron A. Ecdysone and 20 hydroxyecdysone: new hormones for the human parasite schistosoma mansoni. FEBS Lett. 1983;151(2):223-227.

61. Wu W, Tak EY, LoVerde PT. Schistosoma mansoni: SmE78, a nuclear receptor orthologue of Drosophila ecdysone-induced protein 78. Exp Parasitol. 2008;119(2):313-318.

62. Ables ET, Bois KE, Garcia CA, Drummond-Barbosa D. Ecdysone response gene E78 controls ovarian germline stem cell niche formation and follicle survival in Drosophila. Dev Biol. 2015;400(1):33-42.

63. Wu W, Niles EG, El-Sayed N, Berriman M, LoVerde PT. Schistosoma mansoni (Platyhelminthes, Trematoda) nuclear receptors: sixteen new members and a novel subfamily. Gene. 2006;366(2):303-315.

64. Wu W, Niles EG, Hirai H, LoVerde PT. Evolution of a novel subfamily of nuclear receptors with members that each contain two DNA binding domains. BMC Evol Biol. 2007;7:27.

65. Tharp ME, Collins JJ, Newmark PA. A lophotrochozoan-specific nuclear hormone receptor is required for reproductive system development in the planarian. Dev Biol. 2014;396(1):150-157.

66. Förster S, Günthel D, Kiss F, Brehm K. Molecular characterisation of a serum-responsive, DAF-12-like nuclear hormone receptor of the fox-tapeworm Echinococcus multilocularis. J Cell Biochem. 2011;112(6):1630-1642. 